\title{
Novel Approach for Energy Spectrum Probing in Semiconducting Quantum Dots
}

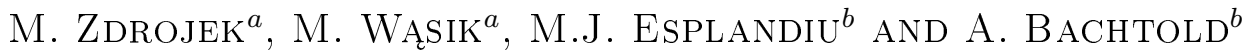 \\ ${ }^{a}$ Faculty of Physics, Warsaw University of Technology, Koszykowa 75, 00-662 Warsaw, Poland \\ ${ }^{b}$ CIN2(CSIC-ICN), Campus UAB, E-08193 Bellaterra, Spain
}

\begin{abstract}
A new technique has been used to probe the electronic properties of quantum dots. Here we discuss the case of semiconducting CdSe dots. This technique allows us to fill (or empty) semiconducting quantum dot with many electrons. The detection scheme is based on an original approach where the investigated particle is attached to only one electrode, a carbon nanotube. The conductance of the nanotube is measured as a function of a gate voltage $\left(V_{\mathrm{g}}\right)$, which allows the detection of individual electrons transferred onto the quantum dot. For certain range of $V_{\mathrm{g}}$ we noticed no electron transfer which is attributed to the energy gap of the CdSe quantum dot. Our study shows that single-electron detection with carbon nanotube transistor represents a new strategy to study the separation between the electronic discrete energy levels of the semiconducting quantum dot.
\end{abstract}

PACS: 73.22.Dj, 73.23.Hk, 73.63.Kv

\section{Introduction}

One of the challenges in molecular electronics is to probe the spectrum properties of quantum systems such as quantum dots (QDs) [1]. To achieve this one needs to access at least one electronic level in such system in order to be able to send current through it. By placing a molecular system between two electrodes, very often it turns out that it is impossible to pass any measurable current through the system, and thus it is impossible to carry out studies on electronic properties. This is one of the biggest obstacles to the development of molecular electronics. Moreover, it is also highly desirable to have possibility to access many electronic levels in the QDs. This would allow to better control the optical and electrical properties of quantum systems. However, the number of electronic levels that can be accessed by current methods [2-5] remains relatively low.

Here we propose novel approach that overcomes these obstacles on the basis of an innovative nanodevice architecture and a novel single electron manipulation scheme. We show a new technique, which enables to investigate the electronic properties of single, highly resistive molecular systems where it is not possible (or extremely difficult) to send measurable currents with conventional methods. The idea is to take advantage of the unique properties of carbon nanotubes to study electronic properties of various types of highly resistive molecular systems. In this paper, as an example we take CdSe QDs.

\section{Samples and experimental setup}

We fabricated devices using standard nanofabrication techniques. Carbon nanotubes were grown by means of chemical vapor deposition (CVD) technique directly on a doped $\mathrm{Si}$ wafer coated with a $1 \mu \mathrm{m}$ thermal $\mathrm{SiO}_{2}$ layer. Single-walled nanotubes (SWNTs) were electrically contacted to two $\mathrm{Cr} / \mathrm{Au}(5 \mathrm{~nm} / 50 \mathrm{~nm})$ electrodes patterned by e-beam lithography. Core-shell CdSe/ZnS quantum dots were employed with an external amine capped long chain hydrocarbon layer. The quantum dots were deposited onto the wafer and were found to be preferentially adsorbed onto nanotubes (Fig. 1a). Eventually, QDs could have been moved by atomic force microscopy (AFM) nearby the nanotube (CNT). Next, the hydrocarbon layer was gently removed, and checked by performing energy dispersive X-ray spectroscopy measurements. Moreover, transmission electron microscopy imaging showed that removal of outer shell of the dot did not modify the crystalline structure of CdSe core and that the diameter is $\approx 5 \mathrm{~nm}$ (inset of Fig. 1a). The back side of the wafer serves as a back gate. See Ref. [6] for more details. Two-point dc transport measurements are conducted at $60 \mathrm{~K}$ in a $\mathrm{He}^{4}$ cryostat (Janis).

\section{Results and discussion}

The charge detection scheme is based on an original approach in which the probed quantum dot is connected only to one electrode, a carbon nanotube (Fig. 1a), in contrast to previous experiments which aimed at contacting individual nanoobjects by at least two electrodes. The carbon nanotube plays two important roles in this nanodevice. At the same time it serves as a reservoir of electrons and it also allows for the detection of single electrons tunneling from the nanotube in order to probe the nanoparticle. The use of only one electrode (nanotube) greatly simplifies the fabrication process of the samples because there is no need to precisely make electrodes separated by a few nanometers (the size of investigated QD). 

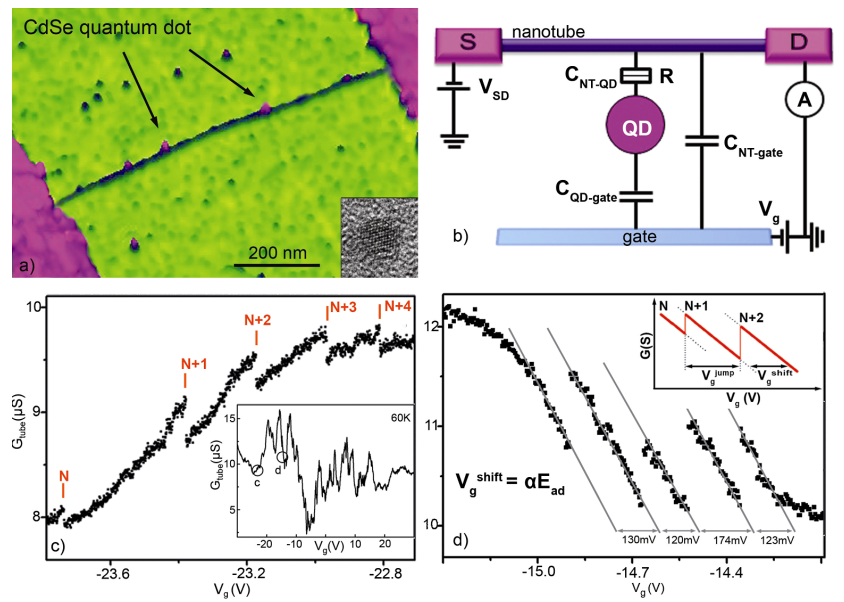

Fig. 1. (a) AFM image of the typical device consisting of carbon nanotube and few CdSe quantum dots. Inset: TEM image of a CdSe QD. (b) Equivalent electrical circuit of our device with three capacitances and a dot-tube tunnel resistance $R$. (c) and (d) $G_{\text {tube }}\left(V_{\mathrm{g}}\right)$ measured in different $V_{\mathrm{g}}$ region at $60 \mathrm{~K}$. The inset in (c) shows $G_{\text {tube }}\left(V_{\mathrm{g}}\right)$ over large $V_{\mathrm{g}}$ scan. Each $G_{\text {tube }}\left(V_{\mathrm{g}}\right)$ shift (marked as red vertical bar) is attributed to a single electron transfer. $V_{\mathrm{g}}^{\text {shift }}$ in (d) fluctuates as the number of transferred electrons is increased, which reflects the fluctuation in the addition energy. Inset in (d) shows the schematic of tube conductance curve as a function of the gate voltage. $V_{\mathrm{g}}^{\text {shift }}$ and $V_{\mathrm{g}}^{\text {jump }}$ characterize the shifts in $V_{\mathrm{g}}$ that is caused by an electron transfer.

The detection of single electrons consists in monitoring the conductance of the carbon nanotube $\left(G_{\text {tube }}\right)$, which is very sensitive to the energy spectrum of the probed nanoobject [7]. The tube conductance is measured as a function of gate voltage (inset of Fig. 1c), which allows to change the Fermi level in an investigated system. The possibility of tuning the Fermi level allows the injection of a large number of electrons. An electron is transferred onto the nanoparticle each time when (during the shift of the Fermi level) an empty state on the nanoobject matches the level of electrochemical potential of the tube. Electron transfer is reflected in the tube conductance as a shift of the conductance along the $V_{\mathrm{g}}$ axis ( $V_{\mathrm{g}}^{\text {shift }}$ ), as it is shown in the inset of Fig. 1d. This shift can be explained in a simple picture: when an extra electron tunnels into the dot, a positive charge has to be accommodated in the nanotube due to the tube-dot capacitance $\left(G_{\text {tube }}\right.$ is proportional to tube charge density) thus making a shift in

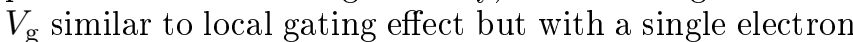
resolution. The second quantity that characterizes the electron transfer is $V_{\mathrm{g}}^{\mathrm{jump}}$ - namely the separation in $V_{\mathrm{g}}$ between two successive electron transfer events. Both $V_{\mathrm{g}}^{\text {shift }}$ and $V_{\mathrm{g}}^{\text {jump }}$ are later used for determination of the energy spectrum of the investigated QD.

Figure 1c shows data in more details. Several electron transfers can be seen, marked by the vertical bars. Each jump in $G_{\text {tube }}$ corresponds to a single electron transfer. The direction of the shift is very important here because it says whether the electron is transferred onto the dot or out of the dot. So, when the electron is tunneling into the dot it is expected to shift the $G_{\text {tube }}\left(V_{\mathrm{g}}\right)$ curve to the right, toward more positive gate voltage values. By counting the number of shifts we can count the number of electrons that were transferred into the dot. Here, it turned out that we can put $\approx 200$ electrons on the CdSe dot. This number of electrons looks considerable big when we compare it with other experiments where people used up to 10 electrons to probe the system [2-5]. In Fig. 1d the values of the shifts of the $G_{\text {tube }}\left(V_{\mathrm{g}}\right)$ curve are not equal, suggesting some fluctuations in the energy between two successive electron levels. This will be discussed later.
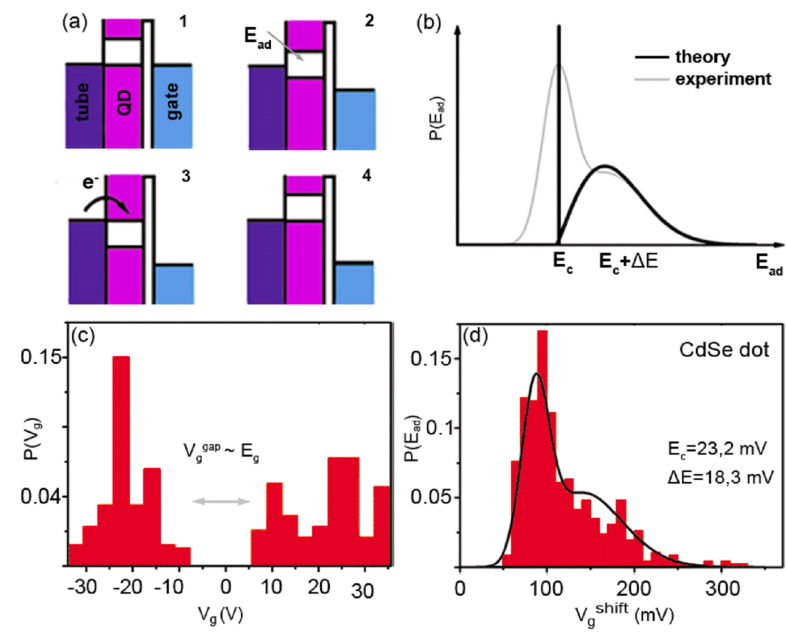

Fig. 2. (a) Schematics of the potentials in the nanotube, the CdSe quantum dot and the gate. For the simplicity, the addition energy equals to the charging energy. (b) Theoretical representation of the bimodal Wigner distribution of the spectrum of a quantum dot (black curve). Gray curve stands for the theoretical prediction of the bimodal Wigner distribution convolved with Gaussian to account for the errors when determining the $V_{\mathrm{g}}^{\text {shift }}$. (c) Normalized histogram of $V_{\mathrm{g}}^{\text {jump }}$ as a function of gate voltage. The gap in $V_{\mathrm{g}}$ reflects the energy gap of semiconducting CdSe dot. (d) Experimental spectrum distribution represented here as normalized histogram of $V_{\mathrm{g}}^{\text {shift }}$. Black curve is the fit with the bimodal distribution convolved with Gaussian to account for experimental errors.

Now, we discuss the relation between the conductance shift and the addition energy. To explain this we take a look on the schematics (Fig. 2a) where the potential in the nanotube, the particle and the gate are shown. At the beginning the potential is set at the same level for tube and dot. As we start to sweep the gate voltage down we also lower the potential of the dot and as soon as an empty level of the dot matches the electrochemical potential of the tube, an electron is transferred onto the particle. This simple picture shows that the change in gate voltage is directly related to the energy that is paid to put an electron onto the system. This can be described 
by the parameter $\alpha$, where $V_{\mathrm{g}}^{\text {shift }}=\alpha E_{\text {ad }}$. The parameter $\alpha$ can be quantified using the electric circuit in Fig. $1 \mathrm{~b}$. We get $\alpha \approx 4$ for the CNT + CdSe device shown in Fig. 1a. See Ref. [6] for details.

The energy between two successive electron levels in the QD (the addition energy) is the sum of the charging energy (the Coulomb repulsion energy) and level spacing energy (confinement energy). When an electron is added onto an empty level, the charging energy $\left(E_{\mathrm{c}}\right)$ needs to be paid, but the addition of a second electron will cost the charging energy and the level spacing energy $(\Delta E)$. This is due to the fact that a level can be filled with two electrons with opposite spins. Quantum mechanically the confinement energy is expected to fluctuate randomly as the number of electrons in the system is increased [8]. This is due to the irregular shape of the system and the chaotic behavior of the electron eigenstates. As a result, the energy spectrum distribution is described by the so-called bimodal Wigner distribution [9] (Fig. 2b).

Figure 2c shows the normalized histogram of the $V_{\mathrm{g}}$ shifts. It is consistent with the theoretical prediction which is here represented by the black fitted curve (bimodal Wigner distribution convoluted with Gaussian to account for the errors when determining the $V_{\mathrm{g}}$ shift). The main peak can be assigned to the charging energy and the long tail is related to the fluctuation of the confinement energy. From Fig. 2d and knowing the parameter $\alpha$, we can obtain the charging energy $(23.3 \mathrm{mV})$ and confinement energy $(18.3 \mathrm{mV})$. Noteworthy, the confinement energy is comparable to the charging energy of the CdSe.

Figure 2c shows the normalized histogram of $V_{\mathrm{g}}^{\text {jump }}$ as a function of gate voltage. We found that electron transfers can be seen everywhere except in the region around zero gate voltage. We attribute this to the energy gap of the semiconducting CdSe particle. Indeed no electron can enter the dot when no free states are available. Let us note that the gap in gate voltage is not the energy gap of the semiconducting QD.

\section{Conclusions}

A new technique, electron counting spectroscopy has been used to probe the electronic properties of quantum dots. Here we discuss the case of semiconducting CdSe dots. This technique allows us to fill or empty semicon- ducting QD with many electrons. The ability to shift the Fermi energy by a large amount holds promise for nanoscale or molecular electronics. The detection scheme is based on an original approach where the investigated particle is attached to only one electrode, a carbon nanotube. The conductance of the nanotube is measured as a function of a gate voltage $\left(V_{\mathrm{g}}\right)$, which allows the detection of individual electrons transferred onto the nano-dot. We noticed no electron transfer for certain range of $V_{\mathrm{g}}$ which is attributed to the energy gap of the CdSe QD. Our study shows that single-electron detection with CNT transistor represents a new strategy to study the separation in energy between the electronic discrete levels of the semiconducting quantum systems.

\section{Acknowledgments}

M.Z. thanks for the support from FNP Homing Plus grant.

\section{References}

[1] e.g. K. Moth-Poulsen, T. Bjornholm, Nature Nano. 4, 551 (2009).

[2] U. Banin, Y. Cao, D. Katz, O. Millo, Nature (London) 400, 542 (1999).

[3] C. Wang, M. Shim, P. Guyot-Sionnest, Science 291, 2390 (2001).

[4] D.L. Klein, R. Roth, A.K.L. Lim, A.P. Alivisatos, P.L. McEuen, Nature (London) 389, 699 (1997).

[5] E.P.A.M. Bakkers, Z. Hens, L.P. Kouwenhoven, L. Gurevich, D. Vanmaekelbergh, Nanotechnology 13 , 258 (2002).

[6] M. Zdrojek, M.J. Esplandiu, A. Barreiro, A. Bachtold, Phys. Rev. Lett. 102, 228604 (2009).

[7] A. Gruneis, M.J. Esplandiu, D. Garcia-Sanchez, A. Bachtold, Nano Lett. 7, 3766 (2007).

[8] L.P. Kouwenhoven, C.M. Marcus, P.L. McEuen, S. Tarucha, R.M. Westervelt, N.S. Wingreen, Mesoscopic Electron Transport, NATO ASI Ser. E, Kluwer, Dordrecht 1997.

[9] M.L. Mehta, Random Matrixes, Academic Press, London 1991. 